Proceedings of the XXIII Conference on Applied Crystallography, Krynica Zdrój, Poland, September 20-24, 2015

\title{
Simple Decoration Model of Icosahedral Quasicrystals in Statistical Approach
}

\author{
R. Strzalka, I. Buganski, J. Wolny* \\ Faculty of Physics and Applied Computer Science, AGH University of Science and Technology, \\ al. A. Mickiewicza 30, 30-059 Krakow, Poland
}

\begin{abstract}
The statistical approach based on the average unit cell concept was recently successfully applied to structural modelling of icosahedral quasicrystals. The structure factor for arbitrarily decorated icosahedral structure was derived for model Ammann tiling (3D Penrose tiling). It is a fully physical-space model where no higher-dimensional description is needed. In the present paper we show the application of the model to the so-called simple decoration scheme - atomic decoration in the nodes, at mid-edges and along body-diagonal of structural units of 3D Penrose tiling. By analyzing the obtained calculated diffraction patterns we show the correctness of the model and its applicability to binary and ternary icosahedral phases.
\end{abstract}

DOI: 10.12693/APhysPolA.130.841

PACS/topics: 61.44.Br, 61.50.Ah

\section{Introduction}

Icosahedral quasicrystals are aperiodic structures with 3D aperiodicity. Their diffraction pattern exhibits full symmetry of an icosahedron, including 5-fold rotation axes. Due to their aperiodicity quasicrystals cannot be modelled as classical (periodic) crystals. The most successful and widely used method of structure modeling is the higher-dimensional analysis [1, 2]. The aperiodic structures (in low dimensions) is lifted to high dimensions where they become periodic. The icosahedral quasicrystals require $6 \mathrm{D}$ space, of which a $3 \mathrm{D}$ subspace, called perpendicular, corresponds to the so-called atomic surfaces (or occupation domains). These 3D objects play a role of multidimensional atoms and by arranging them accordingly in perpendicular space the structural model is obtained [3, 4]. The real (physical) arrangement of atoms is obtained by a projection onto the $3 \mathrm{D}$ physical space, another subspace of the $6 \mathrm{D}$ hyperspace [5-7]. The method has its limitations. The most obvious is the physical interpretation of atomic surfaces, which are just a mathematical construction.

We propose another method of structure modeling of quasicrystals which allows to consider only a $3 \mathrm{D}$ physical space with no need for consideration in high dimensions. Our method, called statistical approach, is based on the concept of the average unit cell (AUC) which is a probability distribution of projections of atoms in physical space onto reference lattices. The lattice constants of the latter are strictly related to the reciprocal space vector used for diffraction peaks indexation and their ratio remains $\tau$ - a golden mean. Details about statistical approach can be found in [8-17].

It is a known characteristics of the icosahedral phases that their atomic structure is cluster-like [18]. In current models all clusters are positioned at nodes of the model quasilattice used for a construction of fully $3 \mathrm{D}$ quasicrystalline setting (called Ammann or 3D Penrose tiling, 3DPT) or its subsets. Pure 3DPT is known to be a good model of $P$-type icosahedral phases $(6 \mathrm{D}$ peri- odic lattice is of the simple cubic or primitive type) [6]. For 3DPT the quasilattice can be spanned by only two structural units, which are acute and obtuse rhombohedra $(\mathrm{AR} / \mathrm{OR})$. The volume ratio of structural units is given by $\tau$, and their facets are golden rhombuses with golden ratio of diagonals lengths. The edge-length $a_{r}$ of the rhombohedra is the quasilattice constant. It can be shown that cluster decoration in cluster-based models can be decomposed into decoration of ARs and ORs [19].

\section{Simple decoration scheme within statistical method}

Recently, the structure factor for arbitrarily decorated 3DPT within the statistical approach was derived $[12,20-22]$. Its correctness was proven for monoatomic decoration (atoms of the same kind in the vertices of the quasilattice only). In this paper we apply the derived structure factor for a simple decoration scheme. The scheme assumes atomic decoration of the structural units in vertices and at mid-edges of the rhombohedra. The AR can be decorated on the body-diagonal with 2 additional atoms (Fig. 1). The simple decoration

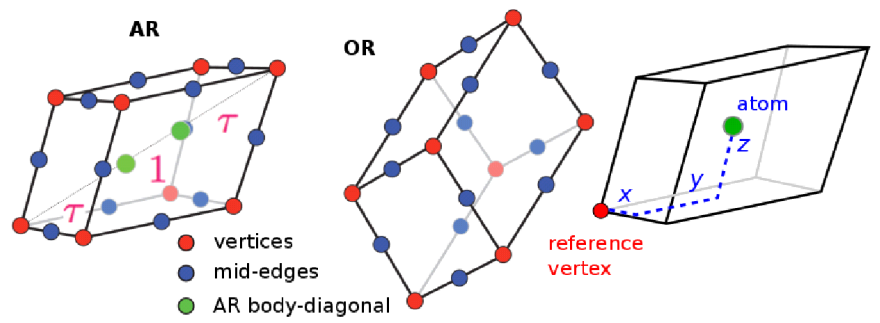

Fig. 1. (a) Structural units of 3DPT: ARs and ORs. Characteristic atomic positions for a simple decoration scheme are presented with red, blue, and green circles. (b) Definition of the atom position $(x, y, z)$ with respect to the reference vertex. Positions of atoms on the AR body diagonal (red circles from part (a)) are: $\boldsymbol{r}_{1}=\left(1 / \tau^{2}, 1 / \tau^{2}, 1 / \tau^{2}\right) \boldsymbol{r}_{2}=(1 / \tau, 1 / \tau, 1 / \tau)$ with respect to reference vertex. 
was first introduced for structural analysis of $\mathrm{Al}-\mathrm{Zn}-$ Mg system [23]. Among other later theoretical models of icosahedral quasicrystals, it has been applied also to binary $\mathrm{Cd}-\mathrm{Yb}$ phase [24]. In this paper we considered two types of purely model decoration schemes of 3DPT (with no partial or mixed occupancies) based on simple decoration:

- triatomic decoration (ternary compounds of $\mathrm{Al}-$ $\mathrm{Pd}-\mathrm{Mn}$ or $\mathrm{Al}-\mathrm{Cu}-\langle\mathrm{Li}, \mathrm{Fe}\rangle$ type): heavy atoms $(\mathrm{Pd} / \mathrm{Cu})$ placed in the vertices, light atoms $(\mathrm{Al})$ at mid-edges and moderate $(\mathrm{Mn} / \mathrm{Fe})$ or $\mathrm{Li}$ atoms on the body-diagonal of AR. From literature we know stable ternary phases $\mathrm{Al}_{70.5} \mathrm{Pd}_{21} \mathrm{Mn}_{8.5}$, $\mathrm{Al}_{60} \mathrm{Cu}_{20} \mathrm{Fe}_{15}$ oraz $\mathrm{Al}_{6} \mathrm{CuLi}_{3}$ [6], while from our previous paper [22] we know that fractions of the characteristic positions (vertex/mid-edge/bodydiagonal) per structural units are 1:3:2. On the top of it we have in average $\tau$-times more ARs than ORs in the 3DPT. The decoration scheme as proposed above appears to be well justified.

- diatomic decoration (binary compound of $\mathrm{Cd}-\mathrm{Yb}$ type): light atoms (Cd) in the vertices and at midedges, heavy atoms $(\mathrm{Yb})$ on the body diagonal of AR. Real atomic composition of the stable $\mathrm{Cd}-\mathrm{Yb}$ phase is $\mathrm{Cd}_{5.7} \mathrm{Yb}$ [24], whereas our model assumes atomic ratio of 4:1 per both structural units.

The structure factor formula requires values of atomic form factors $f_{j}$ for each atom $j$. For simplicity we set values $f_{j}$ independent from scattering vector lengths, and equal to atomic number $Z_{j}$ of the element $j$. This assumption neglects the effect of weakening of the diffraction peaks as the scattering vector length grows, what is the characteristic feature of the X-ray diffraction experiment. However it is known that atomic form factor curves scales with the atomic number [25]. Used values of $f_{j}$-s are shown in Table I.

TABLE I

Values of atomic form factor assumed in the structure factor formula (1) for all elements considered in the model decoration schemes.

\begin{tabular}{c|c|c|c|c|c|c|c|c}
\hline \hline Atom $j$ & $\mathrm{Li}$ & $\mathrm{Al}$ & $\mathrm{Mn}$ & $\mathrm{Fe}$ & $\mathrm{Cu}$ & $\mathrm{Pd}$ & $\mathrm{Cd}$ & $\mathrm{Yb}$ \\
\hline $\begin{array}{c}\text { Atomic form } \\
\text { factor } f_{j}=Z\end{array}$ & 3 & 13 & 25 & 26 & 29 & 46 & 48 & 70
\end{tabular}

To calculate the diffraction pattern we use the following analytic formula for structure factor [22]:

$$
\begin{gathered}
F(\boldsymbol{\chi})=\sum_{l=1}^{10}\left(F(\boldsymbol{\chi})_{l}^{\mathrm{OR}} \sum_{j=1}^{N_{1}} f_{j} \alpha_{j} \mathrm{e}^{\mathrm{i} \boldsymbol{k} \cdot \boldsymbol{r}_{j}}\right) \\
+\sum_{l=1}^{10}\left(F(\boldsymbol{\chi})_{l}^{\mathrm{AR}} \sum_{j=1}^{N_{2}} f_{j} \alpha_{j} \mathrm{e}^{\mathrm{i} \boldsymbol{k} \cdot \boldsymbol{r}_{j}}\right)
\end{gathered}
$$

where $\chi=k_{0}\left[\left(h_{2}-h_{1} \tau\right),\left(h_{4}-h_{3} \tau\right),\left(h_{6}-h_{5} \tau\right)\right], h_{1}-$ $-h_{6}$ - the Miller indices, $N_{1}, N_{2}$ - number of atoms decorating ORs and ARs respectively, $F(\boldsymbol{\chi})_{l}^{\mathrm{OR}}$ Fourier transformation of the distribution of reference vertex for ORs in the AUC, $F(\chi)_{l}^{\mathrm{AR}}-$ Fourier transformation of the distribution of reference vertex for ARs in the $\mathrm{AUC}, f_{j}$ - atomic form factor of $j$-th atom (see Table I), $\alpha_{j}$ - fraction of an atom inside the structure unit (derived in [22]), $r_{j}$ position of the $j$-th atom in the structure unit with respect to the reference vertex (defined as in Fig. 1b).

The successful result of the statistical model of arbitrarily decorated icosahedral quasicrystals is the splitting of the structure factor into two parts: atomic (decoration of structural units with atoms) and lattice (the Fourier transforms $F(\chi)_{l}^{\mathrm{OR}}, F(\chi)_{l}^{\mathrm{AR}}$ of the distributions of structural units at all orientations in the structure). The latter can be calculated before and once for all types of decorations considered. The summation in formula (1) goes from 1 to 10 because we can decompose the AUC into 10 subregions corresponding to 10 independent orientations of ARs and ORs. For details about derivation of the structure factor see [20, 22].

\section{Diffraction pattern and discussion}

Based on the formula (1) we calculated diffraction patterns along arbitrary direction of the scattering vector for five decoration schemes discussed above: $\mathrm{Al}-\mathrm{Cu}-\langle\mathrm{Fe}, \mathrm{Li}\rangle$ , $\mathrm{Al}-\mathrm{Pd}-\mathrm{Mn}, \mathrm{Cd}-\mathrm{Yb}$ and monoatomic decoration with $\mathrm{Al}$ atoms in the vertices only (as a reference diffraction diagram, known as vertex-decoration). Peaks intensities
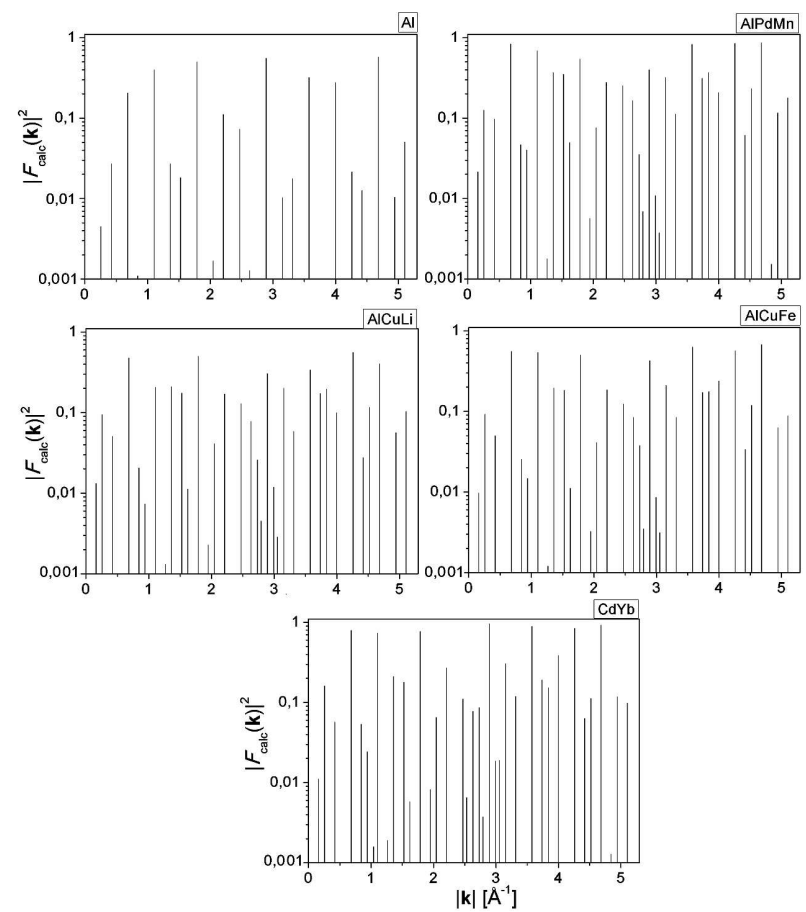

Fig. 2. Diffraction patterns of five simple decorations schemes for 3DPT obtained by statistical approach. Data in semi-logarithmic scale for intensities $>10^{-3}$ reduced to 0-peak height. 
were calculated as $I(|\boldsymbol{k}|)=\left|F_{\text {calc }}(|\boldsymbol{k}|)\right|^{2}$ and rescaled to peaks at $|\boldsymbol{k}|=0$ (0-peaks). The quasilattice constant is chosen to be $a_{r}=4 \AA$, the indices range is $[-5,5]$ and the size of the system is $2 \times 10^{6}$ atoms. Calculated diffraction patterns are presented in Fig. 2. The quantitative analysis of the diffractograms leads us to fundamental conclusions:

(i) Atomic decoration at mid-edges and on bodydiagonal of AR with any atoms significantly influences the diffraction pattern as compared to the reference monoatomic decoration with atoms of the same kind in the vertices. New peaks appear at the positions where there were no previously (or the peaks were weaker than $10^{-3}$ of reduced intensity). More complex atomic structure gives essentially richer pattern in the reciprocal space.

(ii) We observe no systematic extinction of reflections, which is in agreement with known extinction rules for $P$-type quasicrystal (6D Bravais lattice $P \frac{2}{m} \overline{35}$ ). In particular, no weakening of reflections for $\mathrm{Cd}-$ $\mathrm{Yb}$ is observed, although vertices and mid-edges are occupied by the same element.

(iii) Intensities of the most of peaks increased comparing to reference diffraction pattern. In our ternaries and binaries we changed light $\mathrm{Al}$ atoms with much heavier $\mathrm{Cu}, \mathrm{Pd}, \mathrm{Cd}$, which implies the observed increase of the peaks heights. Moreover, some of the peaks (e.g. $\left|\boldsymbol{k}_{1}\right|=0.68 \AA^{-1}$ ) are enhanced largely, which can suggest that heavy atoms $(\mathrm{Cu}, \mathrm{Pd}, \mathrm{Cd})$ contribute significantly to their intensities.

(iv) From a comparison of diffractograms for $\mathrm{Al}-\mathrm{Cu}-$ $\mathrm{Fe}$ and $\mathrm{Al}-\mathrm{Pd}-\mathrm{Mn}$ we conclude that stronger reflections can be observed essentially for the latter case, but not for all peaks equally. Let us focus on the exemplary triple of peaks: $\left|\boldsymbol{k}_{1}\right|=0.68 \AA^{-1}$, $\left|\boldsymbol{k}_{2}\right|=1.1 \AA^{-1},\left|\boldsymbol{k}_{3}\right|=1.79 \AA^{-1}$. The intensity of peak $\left|\boldsymbol{k}_{3}\right|$ almost did not change as compared to $\mathrm{Al}$ vertex-decoration. Most probably the significant contribution to this peak comes from $\mathrm{Al}$ atoms placed at mid-edges. Peak $\left|\boldsymbol{k}_{1}\right|$ is the most changed, it comes probably from heavy atoms in the vertices $(\mathrm{Pd}$ or $\mathrm{Cu})$. The change in intensities of peaks $\left|\boldsymbol{k}_{2}\right|$ is not obvious, this peak most probably comes from several different atoms.

(v) If we compare diffractograms of $\mathrm{Al}-\mathrm{Cu}-\mathrm{Fe}$ and $\mathrm{Al}-$ $\mathrm{Cu}-\mathrm{Li}$ with the reference one, we see pronounced decrease of peak $\left|\boldsymbol{k}_{2}\right|$ in case of decoration with $\mathrm{Li}$ atoms and increase for Fe atom. This brings further interpretation of the origin of peak $\left|\boldsymbol{k}_{2}\right|$, namely it must have a significant contribution from atoms on the body-diagonal of $\mathrm{AR}$ (we know that $f_{\mathrm{Fe}}>$ $\left.f_{\mathrm{Al}}>f_{\mathrm{Li}}\right)$. Similar discussion applies to reflection $\left|\boldsymbol{k}_{5}\right|=4.68 \AA^{-1}$.

(vi) From the diffraction pattern of $\mathrm{Cd}-\mathrm{Yb}$ system we clearly see the dominant height of reflection $\left|\boldsymbol{k}_{4}\right|=$
$2.89 \AA^{-1}$, which is not the case for $\mathrm{Al}-\mathrm{Pd}-\mathrm{Mn}$ or $\mathrm{Al}-\mathrm{Cu}-\mathrm{Fe}$ systems. The contribution to intensity of this peak must come from the heavy $\mathrm{Yb}$ atom (atoms Fe and Mn are clearly lighter). This interpretation can be further confirmed by observation of the diffractogram of $\mathrm{Al}-\mathrm{Cu}-\mathrm{Li}$, where peak $\left|\boldsymbol{k}_{4}\right|$ is strongly decreased.

\section{Summary}

The discussion in (i)-(vi) is only quantitative and cursory, but it brings us to two important conclusions. First, we see that simple decoration scheme of structural units in 3DPT gives a diverse and rather complex diffraction pattern, also for simplified physical parameters. This means that even simple decoration scheme (possibly extended to partial and mixed occupancies of characteristic positions in structural units) can be successfully applied to structural analysis and refinement of icosahedral quasicrystals. Secondly, the use of structure factor (1) obtained by a statistical method gives very realistic diffraction patterns of simple models of icosahedral quasicrystals. This proves its correctness and full applicability to structural modelling of icosahedral phases.

\section{Acknowledgments}

Authors kindly acknowledge the financial support from Polish National Science Centre under grants no. DEC2013/11/B/ST3/03787 and 2014/13/N/ST3/03776. I.B. also acknowledges the support from Marian Smoluchowski Krakow Research Consortium "Matter-EnergyFuture" under KNOW dotation.

\section{References}

[1] M. Duneau, A. Katz, Phys. Rev. Lett. 54, 2688 (1985).

[2] T. Janssen, Acta Crystallogr. A 42, 261 (1987).

[3] M. de Boissieu, H. Takakura, C.P. Gomez, A. Yamamoto, A.P. Tsai, Philos. Mag. 87, 2613 (2007).

[4] A. Katz, D. Gratias, J. Non-Cryst. Solids 153-154, 187 (1993).

[5] A. Yamamoto, Acta Crystallogr. A 52, 509 (1996).

[6] W. Steurer, S. Deloudi, Crystallography of Quasicrystals, Springer-Verlag, Berlin 2009.

[7] J.E.S. Socolar, P.J. Steinhardt, Phys. Rev. B 34, 617 (1986).

[8] J. Wolny, Philos. Mag. A 77, 395 (1998).

[9] P. Buczek, L. Sadun, J. Wolny, Acta Phys. Pol. B 36, 919 (2005).

[10] J. Wolny, A. Wnek, J.L. Verger-Gaugry, J. Comput. Phys. 163, 313 (2000).

[11] B. Kozakowski, J. Wolny, Acta Crystallogr. A 66, 489 (2010).

[12] J. Wolny, B. Kozakowski, P. Kuczera, R. Strzalka, A. Wnek, Isr. J. Chem. 51, 1275 (2011). 
[13] J. Wolny, B. Kozakowski, P. Kuczera, L. Pytlik, R. Strzalka, in: Aperiodic Crystals, Eds. S. Schmidt, R. Withers, R. Lifshitz, Springer, Dordrecht 2013, p. 125.

[14] J. Wolny, B. Kozakowski, P. Kuczera, L. Pytlik, R. Strzałka, A. Wnek, Acta Phys. Pol. A 126, 625 (2014).

[15] J. Wolny, B. Kozakowski, P. Kuczera, L. Pytlik, R. Strzalka, Acta Crystallogr. A 70, 181 (2014).

[16] J. Wolny, P. Kuczera, R. Strzalka, Appl. Phys. Lett. 106, 131905 (2015).

[17] I. Buganski, R. Strzalka, J. Wolny, Phys. Status Solidi B 253, 450 (2016).

[18] H.-C. Jeong, P.J. Steinhardt, Phys. Rev. Lett. 73, 1943 (1994).

[19] M. Baake, U. Grimm, Aperiodic Order, Vol. 1, A Mathematical Invitation, Cambridge University Press, Cambridge 2013.
[20] R. Strzalka, J. Wolny, P. Kuczera, in: Aperiodic Crystals, Eds. S. Schmidt, R. Withers, R. Lifshitz, Springer, Dordrecht 2013, p. 203.

[21] R. Strzalka, J. Wolny, Acta Phys. Pol. A 126, 585 (2014).

[22] R. Strzalka, I. Buganski, J. Wolny, Acta Crystallogr. A 71, 279 (2015).

[23] C.L. Henley, V. Elser, Philos. Mag. B 53, L59 (1986).

[24] H. Takakura, C.P. Gomez, A. Yamamoto, M. de Boissieu, A.P. Tsai, Nature Mater. 6 , 58 (2007).

[25] P.J. Brown, A.G. Fox, E.N. Maslen, M.A. O'Keefe, B.T.M. Willis, in: International Tables for Crystallography, Vol. C, Ed. E. Prince, IUCr, 2006, Ch. 6.1, p. 554 . 